\title{
Bildung in Europa 2010 - Ziele erreicht oder verfehlt?
}

\author{
Jutta Allmendinger \\ Christian Ebner \\ Rita Nikolai
}

Die Veränderungen auf den Arbeitsmärkten und die demografische Entwicklung stellen die Staaten der Europäischen Union (EU) vor große Herausforderungen. Mit der Lissabon-Strategie von 2000 wird Bildung erstmals als zentraler Ansatzpunkt zur Erreichung ökonomischer und sozialer Zielstellungen definiert und damit zu einem entscheidenden Feld der europäischen Beschäftigungsstrategie. Der vorliegende Beitrag analysiert erstens, inwieweit es den EU-Staaten gelungen ist, ihre Leistungsfähigkeit im Bereich der Bildung zu steigern und die von der EU bis 2010 vorgegebenen Zielmarken zu erfüllen. Zweitens wird untersucht, inwieweit sich Konvergenz- und Divergenzprozesse innerhalb der EU in Bezug auf Bildungsergebnisse zeigen. Wächst Europa zusammen und wird es zu einem einheitlichen Bildungsraum?

\section{1 \\ Einleitung}

Seit der Lissabon-Strategie, die im Jahr 2000 von europäischen Staats- und Regierungschefs verabschiedet wurde, verfolgt die Europäische Union (EU) das Ziel, bis 2010 im Hinblick auf Wettbewerbsfähigkeit und wissensbasierte Produkte wie auch Dienstleistungen weltweiter Spitzenreiter zu werden. In diesem Zusammenhang wird der Faktor Bildung erstmalig auch für die Entwicklung von Wirtschaft und Beschäftigung als zentral angesehen. Doch auch angesichts der Veränderungen auf den Arbeitsmärkten und der demografischen Entwicklung gewinnt Bildung an Bedeutung und stellt die europäischen Gesellschaften vor neue Herausforderungen. Nach Projektionen der Vereinten Nationen wird sich nicht nur die Zahl der Europäer im erwerbsfähigen Alter bis zum Jahr 2050 deutlich reduzieren. Vielmehr stellt Europa schon heute die älteste Bevölkerung der Welt - eine Entwicklung, die sich künftig eher verstetigen als verändern wird (UN 2008). Ein kleineres und älteres Europa aber wird - und darin stimmen alle Projektionen überein - Probleme haben, seinen Arbeitsmärkten die benötigten Arbeitskräfte zur Verfügung zu stellen. Derartige Vorzeichen zwingen die EU zum Handeln. Naheliegend wäre die Öffnung europäischer Grenzen, was künftig auf deutlich höhere Beschäftigungsanteile von Nichteuropäern hinauslaufen würde. Auf diese (Gestaltungs-)Möglichkeit stellt sich Europa bisher nicht ein. Die Integrationspolitik ist in fast allen europäischen Ländern defizitär. Umso notwendiger wird damit, den zweiten möglichen Handlungsstrang aufzugreifen: eine veränderte Bildungspolitik, die mehr Personen qualitativ hochwertig bildet, ausbildet und weiterbildet.

$\mathrm{Zu}$ den demografischen Veränderungen treten Veränderungen in den Tätigkeitsanforderungen auf den Arbeitsmärkten von morgen hinzu. Die europäische Wirtschaft durchläuft einen doppelten Strukturwandel. Zum einen ist seit Anfang der 1990er Jahre die Beschäftigungsbilanz des industriellen Sektors deutlich negativ, im Gegensatz zum expandierenden Dienstleistungssektor. Zum anderen expandieren im produzierenden Bereich wie auch innerhalb des Dienstleistungssektors die wissens- und forschungsintensiven Wirtschaftszweige (Belitz et al. 2008). Nicht mehr so sehr die materialorientierte und körperliche Arbeit ist für den Wertschöpfungsprozess bestimmend. Kennzeichen der heutigen Wissensgesellschaft sind vielmehr Fähigkeiten im Umgang mit Informations- und Kommunikationstechniken und ein Metawissen für die Beschaffung, Verwendung und Produktion von Informationen (Rohrbach 2007).

Der technologische Fortschritt geht zulasten von Beschäftigungsmöglichkeiten für geringer qualifizierte Erwerbsper- sonen. Damit wird der Bildungsstand der breiten Bevölkerung zu einer zentralen wettbewerbsrelevanten Ressource. ${ }^{1}$ Zudem führt das steigende Durchschnittsalter der Erwerbsbevölkerung in Verbindung mit

\footnotetext{
Zur Bedeutung von Bildung für die technologische Entwicklung und zum Wachstum von Volkswirtschaften liegt aus der Bildungsökonomie eine breite Literatur vor, auf die nur exemplarisch verwiesen werden kann (Hanushek/Wößmann 2008).
}

Jutta Allmendinger, Prof. Ph.D., Soziologin, Präsidentin des Wissenschaftszentrums Berlin für Sozialforschung (WZB) und Professorin für Bildungssoziologie und Arbeitsmarktforschung an der HumboldtUniversität zu Berlin. Arbeitsschwerpunkte: Soziologie des Arbeitsmarktes, Bildungssoziologie, Soziale Ungleichheit. e-mail: Jutta.Allmendinger@wzb.eu Christian Ebner, Soziologe, Wissenschaftlicher Mitarbeiter in der Projektgruppe "Education and Transitions into the Labour Market" am WZB. Arbeitsschwerpunkte: Bildung und Beschäftigung im internationalen Vergleich. e-mail: Christian.Ebner@wzb.eu Rita Nikolai, Dr., Politologin, Leiterin der Projektgruppe "Education and Transitions into the Labour Market" am WZB. Arbeitsschwerpunkte: Sozialpolitik und Bildungspolitik in Deutschland und im internationalen Vergleich. e-mail: Rita.Nikolai@wzb.eu 
einer sinkenden Halbwertszeit des Wissens dazu, dass eine einzige Bildungsphase am Anfang des Lebens nicht mehr ausreicht und Phasen der Weiterbildung erforderlich werden.

Bildungspolitik ist längst keine nationale Angelegenheit mehr. Zunehmend wird Bildung als Mittel wirtschaftlichen Wohlstands und als Kulturelement internationalisiert. Internationale Organisationen wie die Organisation für wirtschaftliche Zusammenarbeit und Entwicklung (OECD) und die EU haben auf die steigende Bedeutung von Bildung reagiert und sind zu wichtigen Akteuren geworden, die die innenpolitischen Debatten über Reformen nationaler Bildungssysteme maßgeblich geprägt haben. Das Programme for International Student Assessment (PISA) unter Leitung der OECD zielt auf eine international standardisierte Überprüfung von alltags- und berufsrelevanten Fähigkeiten und Kenntnissen 15-jähriger Schülerinnen und Schüler. Mit der PISA-Studie setzt die OECD Standards in der Bildung, die die nationale Politikgestaltung in diesem Feld nachhaltig prägen.

Aufgrund der Bedeutung von Bildung, Ausbildung und Weiterbildung für Wirtschaftswachstum und soziale Integration hat sich auch die EU des Themas Bildung in besonderer Weise angenommen. Im Bereich der Hochschulpolitik ist die EU zur treibenden Kraft der Harmonisierung des europäischen Hochschulraums geworden. Der sogenannte „Bologna-Prozess“ wurde zwar außerhalb des institutionellen Gefüges der EU angestoßen, seit 2001 ist die Europäische Kommission jedoch volles Mitglied in der Bologna-Follow-Up-Group, die als Arbeitsgruppe die Aktivitäten der BolognaMitglieder koordiniert. Zudem gilt die Europäische Kommission als Koordinatorin des Prozesses (Martens/Weymann 2008).

Die bildungspolitischen Aktivitäten der EU erstrecken sich jedoch nicht nur auf den Hochschulbereich. Zunehmend wurde der Bologna-Prozess auch mit anderen bildungspolitischen Aktivitäten der EU verflochten. In seiner Lissaboner Resolution formulierte der Europäische Rat bildungspolitische Ziele, mit denen das europäische Bildungssystem weltweit führend werden soll (COM 2003). Diese verknüpfte der Rat 2002 in seinem Arbeitsprogramm „Allgemeine und berufliche Bildung 2010“ mit konkreten Zielsetzungen und Indikatoren zur Messung der Fortschritte bei der Umsetzung der Ziele.

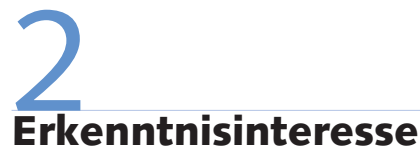

Der zeitliche Bezugsrahmen für das Arbeitsprogramm läuft 2010, also in diesem Jahr, aus. Es ist daher an der Zeit, eine Umsetzungsbilanz der bildungspolitischen Ziele im Rahmen der Lissabon-Strategie zu ziehen. Der vorliegende Beitrag diskutiert, inwieweit es den europäischen Mitgliedstaaten gelungen ist, die von der EU gesetzten Bildungsbenchmarks zu erreichen. Bislang wissen wir wenig darüber, ob der Lissabon-Prozess tatsächlich Wirkung erzielt hat und sich die Leistungen der EU-Staaten im Bereich Bildung seit 2000 verbessert haben. Auch ist wenig darüber bekannt, ob und inwieweit es durch Lissabon zu einer Angleichung der nationalen Bildungsergebnisse gekommen ist. Unser zweites Anliegen ist daher, Konvergenzprozesse der Bildungsergebnisse zwischen den EU-Staaten zu untersuchen.

Zunächst gehen wir auf den LissabonProzess und die formulierten Ziele der EU näher ein (Abschnitt 3). Wir beschreiben dann, welche Entwicklungen die europäischen Länder bereits vollzogen haben. Gibt es Länder, die schon heute die von der EU gesetzten Zielmarken erfüllen? Und welche scheitern im Moment noch gänzlich (Abschnitt 4)? Schließlich interessiert uns, inwieweit wir gemessen an den Bildungsergebnissen von einer Konvergenz nationaler Pfade und einem einheitlichen europäischen Bildungsraum sprechen können (Abschnitt 5). Wir schließen mit einer Zusammenfassung (Abschnitt 6), die das gesellschaftspolitisch relevante Analysepotenzial der Benchmarks verdeutlicht und diskutiert, inwieweit der Aufbau eines europäischen Bildungsraums unterentwickelt bleibt.

\section{3 \\ Bildungspolitik im Rahmen der Lissabon-Strategie}

Aufgrund der großen Aufgaben, die im Bereich von Bildung, Ausbildung und Weiterbildung vor Europa liegen, ist es wichtig, dass sich die EU dieser in besonderer Weise angenommen hat. In ihrer Lissaboner Resolution hat sie bildungspolitische Ziele formuliert, mit denen das europäische
Bildungssystem weltweit führend werden soll (COM 2003). Der Europäische Rat einigte sich 2000 in Lissabon auf das bereits erwähnte und ehrgeizige Ziel, die EU bis 2010 zum wettbewerbsfähigsten und dynamischsten wissensbasierten Wirtschaftsraum der Welt zu machen. Mit der Lissabon-Strategie stärkt der Europäische Rat die Rolle der allgemeinen und der beruflichen Bildung und richtet die europäische Bildungspolitik auf wirtschaftsund beschäftigungspolitische Ziele aus. ${ }^{2}$ Erstmals wird damit Bildung zu einem zentralen Faktor zur Erreichung ökonomischer und sozialer Zielstellungen erhoben (Pépin 2007, S. 121) und ist zugleich eines der zentralen Felder der europäischen Beschäftigungsstrategie (Bektchieva 2004, S. 76). ${ }^{3}$ Die Richtlinien zielen darauf ab, die Mitgliedstaaten dazu zu motivieren, ihre Investitionen in Bildung zu erhöhen, ihre Bildungssysteme zu optimieren und diese als Antwort auf eine wissensbasierte Ökonomie sowie steigende sozioökonomische und demografische Herausforderungen zu modernisieren.

Bezogen auf diese Richtlinien haben sich die europäischen Bildungsministerinnen und -minister auf ihrem gemeinsamen Gipfel in Stockholm im Februar 2001 auf drei Hauptziele geeinigt, die bis 2010 erreicht werden sollen:

- erstens, die Verbesserung der Qualität und Effizienz in den EU-Bildungssystemen;

- zweitens, die Sicherstellung des Zugangs zur Bildung für alle;

- drittens, die globale Öffnung der europäischen Bildungssysteme (COM 2006, S. 4; Dion 2005, S. 302).

Im Zuge dieser Einigung verabschiedete der Europäische Rat 2002 in Barcelona das bildungspolitische Arbeitsprogramm „Allgemeine und berufliche Bildung 2010“, das konkrete Zielsetzungen wie auch Indikatoren zur Messung der Fortschritte bei der Umsetzung der Ziele enthält.

\footnotetext{
2 Mitunter wird die Tendenz zu dieser rein an ökonomischen Kriterien orientierten Bildungspolitik und Fokussierung auf den Arbeitsmarkt kritisiert (Walkenhorst 2008).

3 Für einen Überblick über die Geschichte der europäischen Kooperation in Bildung und Ausbildung siehe detaillierter Pépin (2006); Bektchieva (2004); Becker/Primova (2009); Walkenhorst (2008)
} 
Die drei strategischen Ziele wurden mit 13 Unterzielen (ab 2007 mit 16 Unterzielen) ${ }^{4}$ untermauert und jeweils mit Indikatoren, europäischen Zielmarken (BenchmarkKriterien) und Zeitplänen versehen. Bis 2010 - so die Vereinbarung - sollen die 27 EU-Mitgliedstaaten die folgenden fünf Zielmarken (Benchmarks) erreichen:

(1) Der Anteil der 15-Jährigen mit bedeutenden Leseschwächen soll um mindestens $20 \%$ reduziert werden und damit bei maximal 15,5\% liegen.

(2) Der Anteil der Schulabbrecher soll auf höchstens $10 \%$ reduziert werden.

(3) Wenigstens $85 \%$ der 22-Jährigen sollen die Sekundarstufe II abgeschlossen haben.

(4) Die Zahl der Studienabschlüsse in Mathematik, Naturwissenschaften und Technik (MNT) soll um wenigstens $15 \%$ gesteigert und der Frauenanteil in diesen Fächern bzw. unter den Absolventen soll erhöht werden.

(5) Mindestens 12,5 \% der Bürgerinnen und Bürger sollen lebensbegleitend lernen (COM 2008, S. 10).

Diese Benchmarks sind als europaweite Zielmarken zu verstehen, die als Durchschnittswert von den EU-27-Mitgliedstaaten erreicht werden sollen.

Für die Implementation des Programms einigten sich die europäischen Bildungsministerinnen und -minister in Barcelona auf die Offene Methode der Koordinierung (OMK). Diese Methode wird als ein wichtiges Element der LissabonStrategie angesehen. Denn im Gegensatz etwa zur Wettbewerbspolitik oder der Wirtschafts- und Währungspolitik hat die EU im Bildungsbereich keine Weisungsbefugnisse. D.h. es fehlt ihr im Bildungsbereich an rechtlichen Instrumenten. Mittels der OMK hat die EU jedoch ein politisches Steuerungsinstrument zur Hand, mit dem sie auch ohne Rechtsetzungsbefugnis auf die Politiken der Mitgliedstaaten einwirken kann. Entsprechend entwickelte die Europäische Kommission im Rahmen des Lissabon-Prozesses allgemeine quantitative Ziele, ohne aber vorzuschreiben, auf welchem Wege die Mitgliedstaaten die Ziele erreichen sollen. Die Koordinierungsmethode lässt den EU-Mitgliedstaaten den Freiraum, selbst zu entscheiden, wie die je- weiligen Ziele erreicht werden. Durch den Austausch von Good-Practice-Beispielen sowie regelmäßiges Monitoring werden die Fortschritte überprüft und die Ergebnisse der einzelnen Mitgliedstaaten untereinander verglichen (Pépin 2007, S. 128; Dion 2005, S. 299).

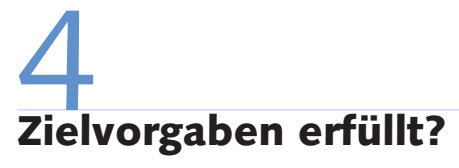

Es ist offensichtlich, dass hinter den in Lissabon gesetzten Zielen gängige Maße zur Messung von Bildung stehen. Kompetenzmessungen (Benchmark 1) gehen Hand in Hand mit Ansätzen, die den Schulbesuch und erreichte Schulabschlüsse erfassen (Benchmarks 2 und 3). Daneben werden Indikatoren erstellt, welche die fachspezifische Ausrichtung des Bildungssystems auf Herausforderungen des technologischen Wandels messen sollen (Benchmark 4). Ebenso wird die Notwendigkeit lebenslangen Lernens (Benchmark 5) betont.

Wichtige Entwicklungstendenzen der Bildungssysteme Westeuropas sind nach 1945 gemäß Walter Müller et al. (1997, S. 178) $)^{5}$ eine längere Verweildauer im Bildungssystem und eine höhere Bildungsbeteiligung für breite Bevölkerungsschichten. Gymnasien und höhere Sekundarschulen wurden für weitere Bevölkerungsteile geöffnet, und auch die Teilhabe an Tertiärbildung verlor ihren bis dahin weitgehend exklusiven Charakter. Diese Bildungsexpansion hat sich bis zum heutigen Zeitpunkt fortgesetzt - es bestehen dennoch große Unterschiede bezüglich Niveau und Veränderung der Bildungsteilhabe zwischen den europäischen Staaten. Inwieweit die europäischen Staaten seit Lissabon ihre Leistungsfähigkeit im Bereich der Bildung steigern konnten und die seitens der EU gesetzten Benchmarks erfüllen, wird im Folgenden diskutiert.

Bildung lässt sich einerseits über die Dauer des Schulbesuchs bzw. anhand von Zertifikaten, d.h. durch den höchsten erreichten Abschluss in Schule und Ausbildung, messen. Seit Kurzem stehen der empirischen Bildungsforschung weitere Maßzahlen zur Verfügung, und zwar Maße zur Messung kognitiver Kompetenzen. Am bekanntesten ist die bereits erwähnte PISA-Studie, die international breit ansetzend Kompetenzen von 15-jährigen Schülerinnen und Schülern in Lesen, Mathematik und Naturwissenschaften misst. „Bildungsarmut“ (Allmendinger 1999) tritt zum einen auf, wenn Personen ein bestimmtes Kompetenzniveau unterschreiten (Kompetenzarmut). Im Falle der PISA-Studien werden Schülerinnen und Schüler als absolut kompetenzarm bezeichnet, wenn sie unter Kompetenzstufe II liegen und damit ernsthafte Schwierigkeiten aufweisen, mit schriftlicher Information umzugehen. Bildungsarmut liegt zum zweiten vor, wenn Personen über keinen relevanten Abschluss verfügen (Zertifikatsarmut). Studien zeigen, dass Personen ohne Sekundarstufe-II-Abschluss ${ }^{6}$ in Europa mit extremen Schwierigkeiten auf dem Arbeitsmarkt konfrontiert sind (OECD 2009; European Commission 2008). Beide Maße, Kompetenzarmut und Zertifikatsarmut als Ausprägungen der Dimension Bildungsarmut, sind Schwerpunkt der ersten beiden EU-Benchmarks.

Die Zielvorgaben der EU für das Jahr 2010 beziehen sich auf die EU-27-Staaten. Um die Entwicklung im Zeitverlauf darzustellen, ziehen wir bei allen Indikatoren auch den Durchschnitt der EU-15-Staaten hinzu, da für diese Ländergruppe durchgängig Daten seit 2000 vorliegen. ${ }^{7}$

\subsection{BENCHMARK 1: ABBAU VON KOMPETENZARMUT}

Abbildung 1 zeigt Niveau und Entwicklung der Kompetenzarmut (Benchmark 1) für die europäischen Staaten. Betrachtet werden die Jahre 2000, 2003 sowie 2006, und damit alle drei Erhebungszeitpunkte der PISA-Studien. Bezogen auf die Lesekompetenzen ist das angelegte EU-Ziel

$4 \quad$ Zu den Unterzielen siehe COM (2008, S. 10).

5 Zum Ausbau der Massenbildung siehe auch Boli et al. (1985).

6 Im Falle Deutschlands fallen hierunter Personen, die weder über ein Abitur verfügen noch eine berufliche Ausbildung abgeschlossen haben.

7 Eurostat wendet zur Klassifikation von Bildungsabschlüssen die Internationale Standardklassifikation ISCED-97 an. Die von Eurostat ausgewiesenen Daten beruhen auf Angaben der jeweiligen nationalen Statistikämter und Ministerien. Die Daten weisen zum Teil Probleme von Validität und Reliabilität auf. Im Rahmen der ISCED-Klassifikation werden in machen Ländern anstelle von Bildungsabschlüssen die Bildungsbeteiligungen erhoben. Auch eine klare Unterscheidung zwischen Abschlüssen des Sekundarbereichs II und dem postsekundären, nicht-tertiären Bereich ist in vielen Ländern nicht möglich. Teilweise werden auch berufsbildende Qualifikationen nicht ausreichend berücksichtigt und es fehlen klare Richtlinien zum Umgang mit Schul-, Ausbildungs- und Studienabbrechern (Schneider 2008). 


\section{Abb. 1: Kompetenzarmut - Anteile von Schülerinnen und Schülern unterhalb Kompetenzstufe II im Lesen (2000, 2003 und 2006) - in \% -}

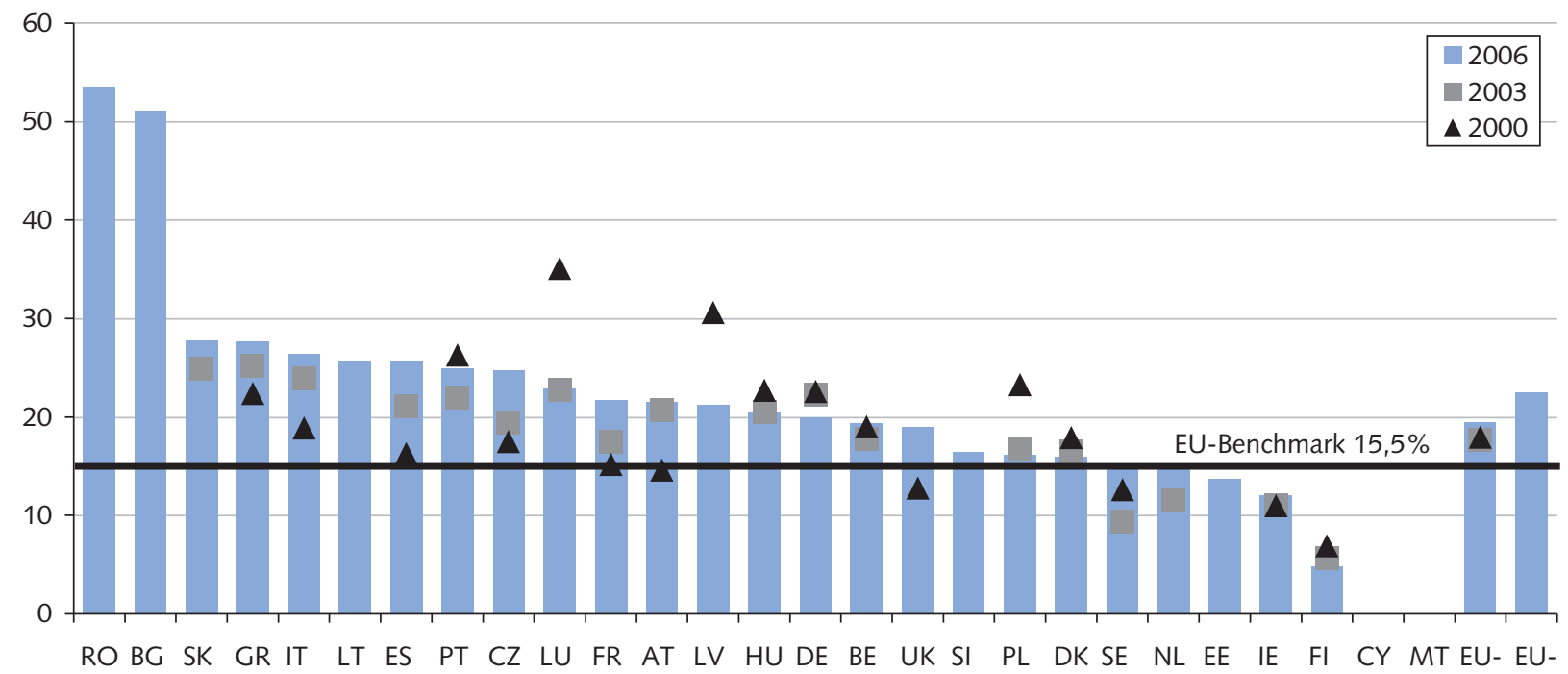

Anmerkungen: EU-15 (2000 ohne Niederlande); EU-15 (2003 ohne Großbritannien).

bescheiden. Die Kommission akzeptiert einen Anteil von 15,5\% der 15-jährigen Schülerinnen und Schüler unterhalb der „Armutsgrenze“ (Kompetenzstufe II). Dennoch erfüllen nur fünf der 25 getesteten EU-Staaten ${ }^{8}$ im Jahr 2006 den Zielwert: Finnland deutlich, aber auch Irland, Estland, die Niederlande und Schweden. Alle anderen Länder liegen darüber, d.h. sie haben das Ziel verfehlt, wenn im Fall von Dänemark, Polen und Slowenien auch nur knapp. Anteile von kompetenzarmen Schülerinnen und Schülern von sogar über $50 \%$ haben Rumänien und Bulgarien, über einem Wert von $25 \%$ liegen die Slowakei, Griechenland, Italien, Litauen und Spanien. Betrachtet man ausschließlich die Entwicklung des EU-15-Länderdurchschnitts im selben Zeitraum, ist keine Verringerung der Kompetenzarmut zu erkennen, tendenziell sogar ein leichter Anstieg: Betrug der Mittelwert im Jahr 2000 18,0 \%, beläuft sich dieser 2006 auf 19,5\%. Der Durchschnittswert für die EU-27-Staaten lag im Jahr 2006 gar bei $22,5 \%$. Warum in Ländern wie etwa Italien, Frankreich, Österreich oder im Vereinigten Königreich Kompetenzarmut seit 2000 deutlich zugenommen hat - was ein alarmierendes Ergebnis ist -, kann aus dem bisherigen Forschungsstand nicht beantwortet werden. Bis zur vierten PISA Erhebung 2009, deren Ergebnisse jedoch erst im Winter 2010 vorliegen werden, hätten die EU-Staaten die Kompetenzarmut eu- ropaweit um sieben Prozentpunkte senken müssen, um die Zielvereinbarung von 15,5 \% erreichen zu können.

\subsection{BENCHMARK 2 UND 3: SCHUL- BESUCH UND BILDUNGSZERTIFIKATE}

Wie wichtig die Reduzierung von Bildungsarmut für alle Mitgliedstaaten ist, wird auch durch die Tatsache unterstrichen, dass die Kommission einen zweiten Maßstab von Bildungsarmut anführt: das Fehlen von Bildungszertifikaten. Das explizite Ziel von nur noch $10 \%$ „Schulabbrechern" (Benchmark 2) wurde dafür festgelegt. Als Schulabbrecher gelten all diejenigen 18- bis 24-Jährigen, die keinen Sekundarstufe-II-Abschluss erworben haben und sich auch nicht mehr im Bildungssystem befinden. Heutzutage sind junge Menschen, die die Schule ohne Abschluss der Sekundarstufe II verlassen, mit hohen Arbeitsmarktrisiken konfrontiert. Abbildung 2 gibt Aufschluss über Benchmark 2.

Im Jahr 2008 erfüllten sechs Länder der EU-27 die gesetzte Zielmarke: Polen, Slowenien, die Tschechische Republik, die Slowakei, Litauen und Finnland; Österreich verfehlt nur knapp. Insbesondere in drei der europäischen Länder - Malta, Portugal und Spanien - ist der Anteil der Zertifikatsarmen mit jeweils über $30 \%$ besonders hoch. Es wird an dieser Stelle klar, dass die Länder mit der höchsten Zertifikatsar- mut nicht zwangsweise diejenigen mit der höchsten Kompetenzarmut sein müssen. ${ }^{9}$ Es erscheint vor diesem Hintergrund mehr als sinnvoll, für die Messung von Bildungsarmut beide Indikatoren auszuweisen, also Zertifikatsarmut und Kompetenzarmut. Betrachtet man die Entwicklung des EU15-Länderdurchschnitts über die Zeit, ist nur eine leichte Verbesserung zu erkennen. Im Jahr 2000 lag diese bei 19,3 \%, 2008 „nur“ noch bei 16,7 \%. Für die gesamte EU-27 liegt der Durchschnittswert etwas besser, nämlich bei $14,9 \%$. Bis 2010 müssten die EU-27-Staaten also den Anteil von Zertifikatsarmen in nur zwei Jahren um weitere 4,9 Prozentpunkte gesenkt haben, um die Zielvorgabe von $10 \%$ zu erfüllen. Auf Basis der bisherigen schleppenden Entwicklung ist dies nicht zu erwarten.

Der dritte Benchmark stellt die Logik von Zertifikatsarmut quasi ,auf den Kopf “. Die Zielvorgabe ist diesmal, dass wenigstens 85 \% der 22-Jährigen die Sekundarstufe II abgeschlossen haben sollen. Anders ausgedrückt: Nur $15 \%$ der 22-Jährigen sollen ohne einen Abschluss der Sekundarstufe II verbleiben. Ob sich die 22-Jährigen im Bildungssystem befinden, ist aber im Gegensatz zu Benchmark 2 nicht von Relevanz.

8 Zwei der EU-27-Staaten, Malta und Zypern, haben an keiner der drei PISA-Erhebungen teilgenommen, sodass hier keine Werte vorliegen.

9 Eine ausführlichere Diskussion zu diesem Zusammenhang findet sich in Allmendinger et al. (2010). 
Abb. 2: Frühzeitige Schulabgänger* (2000, 2003 und 2008) - in \% -

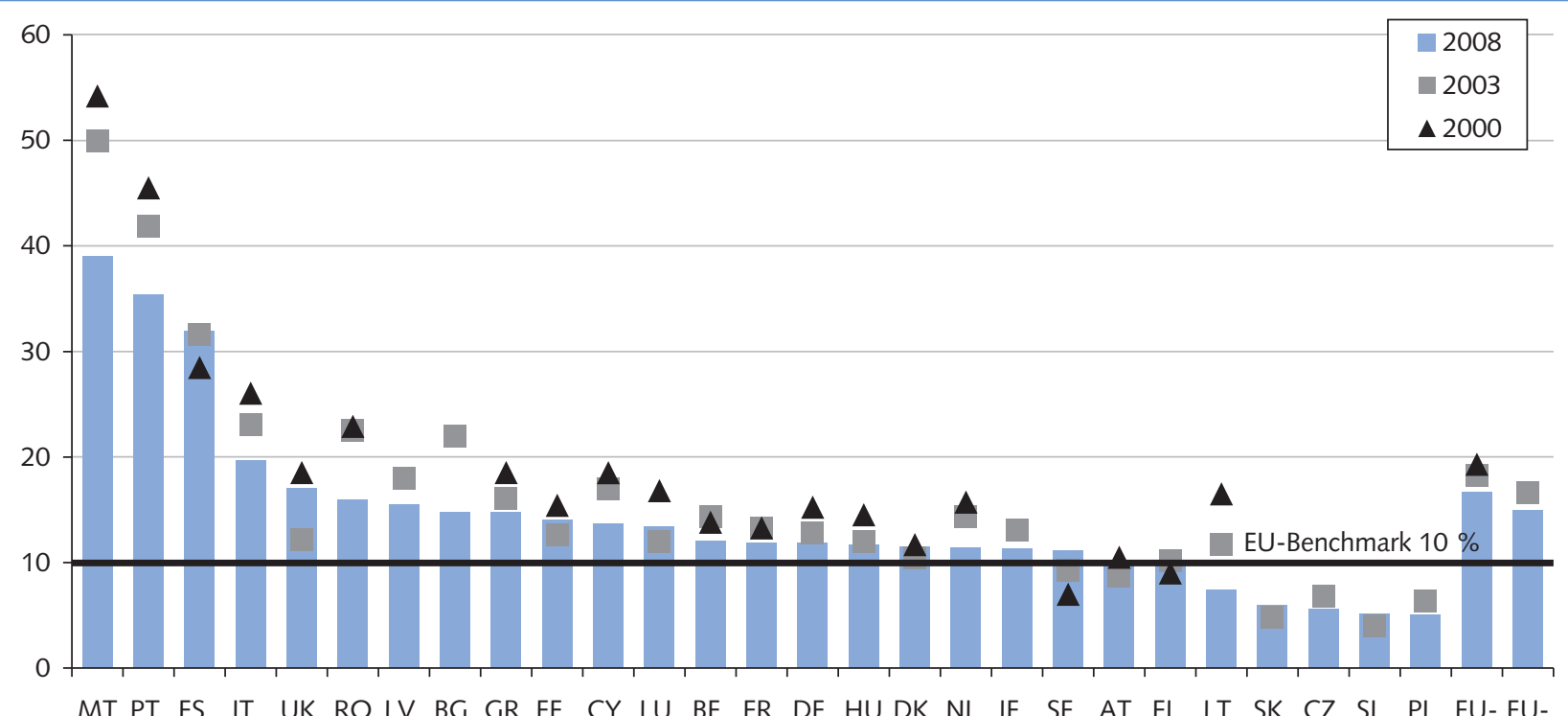

1527

*18-24 Jährige, höchster Bildungsabschluss Sekundarstufe I, keine Teilnahme an Aus- und Weiterbildungsmaßnahmen. EU-15 (2000 ohne Irland).

Da es zu Problemen aufgrund zu kleiner Stichprobengrößen kommen kann, zieht die EU zudem die Alterskategorie 20 bis 24 Jahre anstelle der 22-Jährigen heran. Die Länderergebnisse ähneln dem vorigen Benchmark: Die Slowakei, die Tschechische Republik, Polen, Slowenien, Litauen, Schweden, Irland, Finnland haben die Zielvorgaben bereits 2008 erreicht, Malta, Portugal und Spanien sind im selben Jahr von der Zielerreichung noch weit entfernt. Der EU-15-Durchschnitt hat sich von $73,7 \%$ (2000) auf 75,8 \% (2008) geringfügig verbessert, der EU-27-Durchschnitt lag 2008 bei 78,5\%. Seit 2000 haben sich die EU-27-Staaten von 76,6 \% um rund 1,9 Prozentpunkte hocharbeiten können, was einer jährlichen Steigerung von rund 0,2 Prozentpunkten entspricht. Damit war aber bereits im Jahr 2008 klar: Um bis heute (2010) die Zielmarke von $85 \%$ zu erreichen, hätten die EU-27-Staaten den Anteil von Personen mit mindestens einem Abschluss der Sekundarstufe II um 6,5 Prozentpunkte gegenüber 2008 steigern müssen, also um einen Wert, der angesichts der Erfahrungen mit den bisherigen Entwicklungspotenzialen als unrealistisch erscheinen muss.

\subsection{BENCHMARK 4: HOCHSCHUL- ABSCHLÜSSE IN MNT}

In den Bereichen Mathematik, Naturwissenschaften und Technik (MNT) herrscht zum Teil schon heute Fachkräftemangel. Dabei sind es genau diese Berufsbereiche, die für Innovationen und Wirtschaftswachstum von Volkswirtschaften zentral sind (EFI 2009). Der vierte Benchmark zielt daher darauf ab, die absolute Anzahl der Hochschulabsolventinnen und -absolventen in MNT von 2000 bis 2010 um wenigstens $15 \%$ zu erhöhen. Im Jahr 2000 gab es innerhalb der EU-27 675.600 Absolventinnen und Absolventen in diesen Fachbereichen, im Jahr 2007 869.100. ${ }^{10}$ Damit war bereits 2007 ein Zuwachs von 28,6\% zu verzeichnen, der den Benchmark deutlich erfüllt. Allerdings: Der zugrunde liegende Indikator birgt Tücken, da er nicht gewichtet, also ins Verhältnis zur Bevölkerungsentwicklung gesetzt wird. So kann sich etwa die absolute Zahl der Absolventen allein aufgrund demografischer Schwankungen, zum Beispiel durch ein kohortenspezifisches Anwachsen der Bevölkerungszahl, erhöht haben. Es bietet sich daher eher an, die Absolventinnen und Absolventen in MNT pro $1.000 \mathrm{der}$ Bevölkerung im Alter von 20 bis 29 Jahren zu untersuchen. Die Daten dazu sind beim Europäischen Amt für Statistik (Eurostat) erhältlich. Im Abschnitt 5 wird dieser Indikator berücksichtigt.

Ein weiteres Ziel der EU ist, den Anteil von Frauen in MNT zu erhöhen. Der Anteil der weiblichen MNT-Absolventen nahm im EU-15-Durchschnitt von 29,4\% auf 30,6 \% im Jahr 2006 zu, der EU-27Durchschnitt lag 2006 bei 31,6 \%. Bei der Annäherung an ein ausgewogenes Verhältnis von Frauen und Männern schneiden Estland, Bulgarien, Griechenland und Portugal am besten ab (COM 2008, S. 79).

\subsection{BENCHMARK 5: LEBENSBE- GLEITENDES LERNEN}

Die insgesamt alternde Erwerbsbevölkerung Europas und die Dynamik der Märkte hat die Europäische Kommission dazu veranlasst, eine Zielvorgabe für das lebensbegleitende Lernen ${ }^{11} \mathrm{zu}$ formulieren. Die Vorgabe liegt bei einer Beteiligung von 12,5\% für die Bevölkerung im Alter von 25 bis 64 Jahren. Weiterbildungen wurden 2008 (Zeitpunkt der Datenerhebung) vor allem in Dänemark, Finnland, Schweden, also den skandinavischen Ländern, wahrgenommen, aber auch Großbritannien, Niederlande, Slowenien und Österreich liegen über der Zielvorgabe von 12,5 \%. Besonders wenig Weiterbildung findet bisher in

10 Da für Griechenland für 2000 keine Zahlen von Eurostat ausgewiesen werden, wird Griechenland auch 2007 nicht berücksichtigt.

11 Laut Definition der Europäischen Union umfasst lebensbegleitendes Lernen sowohl formale wie auch nicht-formale Lernaktivitäten. Die Europäische Arbeitskräfteerhebung erfasst die Teilnahme an Aus- und Weiterbildungsmaßnahmen innerhalb der letzten vier Wochen vor der Umfrage. 
Bulgarien, Rumänien, Griechenland, Ungarn und der Slowakei statt. Die EU-15 haben sich dabei langsam von 8,0 \% (2000) auf $10,9 \%$ (2008) gesteigert. Betrachtet man die gesamte EU-27 liegt der Wert 2008 bei $9,5 \%$. Hätten die EU-27-Staaten das bis 2010 gesetzte Ziel von 12,5\% erreichen wollen, dann hätten sie in nur zwei Jahren ihre Anstrengungen um weitere drei Prozentpunkte steigern müssen. Die bisherige Entwicklung - die Werte stagnieren seit 2004 - deutet hierauf nicht hin.

\section{5 \\ Konvergenz- und Diver- genzprozesse}

Der Blick auf den Kalender zwingt zu folgender Bilanz: Die europäischen Staaten sind von den für 2010 durch die EU selbst gesetzten Zielvorgaben weit entfernt. Damit stellt sich die zweite Frage: Wie ist es um die EU-internen Angleichungsprozesse in Bezug auf Bildungschancen und Bildungsergebnisse bestellt? Dieser Aspekt erscheint auch vor dem Hintergrund wichtig, dass innereuropäische Arbeitsmarkt- und Bildungsmobilität gefördert werden soll. Zeichnet sich entlang der bereits beschriebenen Ziele die Entstehung eines gemeinsamen europäischen Bildungsraums ab?

Tabelle 1 weist statistische Maßzahlen aus, mit deren Hilfe untersucht werden kann, ob es im Hinblick auf die einzelnen Indikatoren zu Konvergenz- oder Divergenzprozessen innerhalb der EU gekommen ist. Nicht für alle EU-27-Staaten liegen Daten für den Ausgangszeitpunkt des Vergleichs (2000) vor. Daher konzentriert sich unsere Betrachtung und Interpretation von möglichen Konvergenz- oder Divergenzprozessen auf den Durchschnitt der EU-15-Staaten, für die die notwendigen Angaben vorhanden sind ( $\mathrm{Ta}$ belle 1, grau hinterlegt). Die Maßzahl zu MNT bildet die Absolventinnen und Absolventen in MNT pro 1.000 der Bevölkerung im Alter von 20 bis 29 Jahren ab und berücksichtigt somit - im Gegensatz zum ursprünglichen EU-Benchmark-auch die unterschiedlichen Bevölkerungszahlen der EU-Mitgliedsländer.

Während der Mittelwert ausweist, auf welchem Niveau sich Europa bezogen auf die einzelnen Indikatoren befindet, zeigt die Spannweite die Differenz zwischen dem niedrigsten und dem höchsten Wert, den die EU-Staaten aufweisen. Die Standardabweichung bildet $\mathrm{ab}$, wie sehr die

\begin{tabular}{|c|c|c|c|c|c|c|c|}
\hline & & \multicolumn{2}{|c|}{ Mittelwert } & \multicolumn{2}{|c|}{ Spannweite } & \multicolumn{2}{|c|}{$\begin{array}{l}\text { Standard- } \\
\text { abweichung }\end{array}$} \\
\hline & & EU-15 & EU-27 & EU-15 & EU-27 & EU-15 & EU-27 \\
\hline \multirow{3}{*}{$\begin{array}{l}\text { Kompetenzarmut: } \\
\text { Lesekompetenz }(\%)^{1}\end{array}$} & 2000 & 18,0 & - & 28,2 & - & 7,1 & - \\
\hline & 2006 & 19,5 & 22,5 & 22,9 & 48,7 & 6,1 & 10,5 \\
\hline & Diff. 2006-2000 & 1,5 & - & $-5,3$ & - & $-1,0$ & - \\
\hline \multirow{3}{*}{$\begin{array}{l}\text { Zertifikatsarmut: } \\
\text { Schulabbrecher }(\%)^{2}\end{array}$} & 2000 & 19,3 & - & 36,3 & - & 9,5 & - \\
\hline & 2008 & 16,7 & 14,9 & 25,6 & 34,0 & 7,8 & 8,5 \\
\hline & Diff. 2008-2000 & $-2,6$ & - & $-10,7$ & - & $-1,7$ & - \\
\hline \multirow{3}{*}{$\begin{array}{l}\text { Zertifikate: } \\
\text { mind. SEK II }(\%)^{2}\end{array}$} & 2000 & 73,7 & - & 44,5 & - & 10,9 & - \\
\hline & 2008 & 75,8 & 78,5 & 33,6 & 39,3 & 9,8 & 10,5 \\
\hline & Diff. 2008-2000 & 2,1 & - & $-10,9$ & - & $-1,2$ & - \\
\hline \multirow{3}{*}{$\begin{array}{l}\text { Teilnahme an } \\
\text { Weiterbildung }(\%)^{2}\end{array}$} & 2000 & 8,0 & - & 20,6 & - & 7,5 & - \\
\hline & 2008 & 10,9 & 9,5 & 27,3 & 28,8 & 7,9 & 6,9 \\
\hline & Diff. 2008-2000 & 2,9 & - & 6,7 & - & 0,4 & - \\
\hline \multirow{3}{*}{$\begin{array}{l}\text { MNT-Absolventen pro } \\
1000 \text { d. } 20-29 \mathrm{J.}^{3}\end{array}$} & 2000 & 11,9 & - & 18,5 & - & 5,9 & - \\
\hline & 2007 & 14,4 & 12,1 & 12,2 & 16,5 & 4,0 & 4,2 \\
\hline & Diff. 2007-2000 & 2,5 & - & $-6,3$ & - & $-1,9$ & - \\
\hline \multicolumn{8}{|c|}{$\begin{array}{l}\text { Quellen: 1) OECD 2001, 2007; - 2) Eurostat-Online unter http://nui.epp.eurostat.ec.europa.eu/nui/show.do?dataset=Ifsi_ } \\
\text { edu_a\&lang=de [Zugriff am 22.01.2010]; - 3) Eurostat-Online unter http://nui.epp.eurostat.ec.europa.eu/nui/show. } \\
\text { do?dataset=educ_thflds\&lang=de [Zugriff am 22.01.2010]; Für den Indikator zu den } \\
\text { MNT-Absolventen werden die statistischen Maßzahlen stets ohne Luxemburg berechnet, } \\
\text { da Eurostat nur für das Jahr } 2000 \text { einen Wert ausweist. }\end{array}$} \\
\hline
\end{tabular}

Untersuchungsländer im Durchschnitt um den Mittelwert streuen.

Konvergenzprozesse zeigen sich gleich bei vier der fünf Indikatoren. Spannweite und Standardabweichung nehmen für die EU-15 im Hinblick auf Kompetenzarmut, den Anteil der Schulabbrecher, den Anteil von Absolventen der Sekundarstufe II und die Studienabsolventen in MNT ab. In Bezug auf den Indikator „Weiterbildung“ dagegen nehmen die Unterschiede zwischen den EU-15-Staaten zu, die Länder werden sich über die Zeit also immer unähnlicher.

Im Hinblick auf die gesamten EU-27Staaten lassen sich noch keine Aussagen bezüglich längerfristiger Entwicklungen treffen, da Daten für alle Mitgliedstaaten erst seit Mitte des letzten Jahrzehnts vorliegen. Gemessen an der Standardabweichung unterscheidet sich die Streuung der Werte zwischen den EU-15- und EU-27-Staaten vor allem bei der Lesekompetenz: Mit der Einbindung der neuen Mitgliedstaaten in die EU geht erhebliche Heterogenität einher. Hinsichtlich Bildungszertifikaten, Weiterbildungsverhalten und MNT-Absolventen ist die Streuung für die EU-15- und EU-27-Staaten dagegen vergleichbar.

Alles in allem sind für die alten Mitgliedstaaten bei vier Indikatoren leichte Konvergenzprozesse zu beobachten. Von einem einheitlichen Bildungsraum lässt sich jedoch noch lange nicht sprechen; weder bei Betrachtung der EU-15- noch der EU-27-Staaten.
Konvergenz steht zudem nicht in unmittelbarer Verbindung mit der Qualität von Bildungsergebnissen. So nimmt der Anteil kompetenzarmer Schülerinnen und Schüler über die Zeit zu, auch wenn die Unterschiede zwischen den Ländern sinken. Auf der anderen Seite sinkt der Anteil zertifikatsarmer Schülerinnen und Schüler im Zuge der Angleichung europäischer Verhältnisse. Dass ein Anstieg bei der Kompetenzarmut mit einem Rückgang von Zertifikatsarmut einhergeht, wirft die Frage nach dem sich verändernden (Signal-)Wert von Zertifikaten auf, eine Entwicklung, die in diesem Artikel nicht diskutiert werden kann. Weiter unterstreicht dieser Befund, dass es zur Messung von Bildungsarmut in der Tat beide Maße, also Zertifikats- und Kompetenzarmut, braucht, denn deren Entwicklung verläuft voneinander unabhängig.

\section{Fazit}

Die Lissabon-Zielsetzung eines wissensbasierten Wirtschaftsraumes, die demografische Entwicklung in Europa und veränderte Anforderungen auf den Arbeitsmärkten von morgen setzen die Systeme von Bildung, Ausbildung und Weiterbildung unter Druck. Die meisten europäischen Länder wissen, dass es höchste Zeit zum Handeln ist. Bildungsreserven müssen besser genutzt 
und erreichte Bildungsniveaus durch kontinuierliche Weiterbildung besser bewahrt werden, will Europa und wollen die einzelnen europäischen Länder in Zukunft wettbewerbsfähig bleiben. Die Herausforderung an die europäischen Bildungssysteme besteht jedoch nicht nur in der Bereitstellung von qualifizierten Arbeitskräften. Bildung ist auch ein Motor für soziale Integration. Die EU-Mitgliedstaaten sollten daher nicht nur bestrebt sein, ein hohes durchschnittliches Leistungsniveau zu erzielen, sondern dieses auch für einen möglichst großen Teil der Bevölkerung zu erreichen. Eine gute Schul- und Berufsausbildung ist ein nachhaltiges Instrument, um die gesellschaftliche Teilhabe der Menschen in ganz Europa überhaupt erst zu ermöglichen.

Das Beobachtungsfenster im vorliegenden Beitrag ist zwar klein und ein abschließendes Urteil zum Lissabon-Prozess kann an dieser Stelle noch nicht gefällt werden. Jedoch können in dem kurzen Zeitraum seit 2000 einige Trends zur Entwicklung im europäischen Bildungsraum beobachtet werden. Das heißt zunächst: Insgesamt ist die bisherige Bestandsaufnahme ernüchternd. Nur eine Minderheit der Mitgliedsländer erreicht bislang die für das Jahr 2010 gesetzten Ziele. Lediglich Finnland und Schweden schneiden fast durchgängig zufriedenstellend ab. Länder, die einen niedrigen Anteil von Schulabbrechern aufweisen, sind nicht unbedingt gleichzeitig die Länder, die auch einen niedrigen Anteil von Kompetenzarmen haben.

Gemessen an Streuungsmaßen wie der Spannweite und der Standardabweichung sind sich die europäischen Staaten zwar hinsichtlich vier der fünf Indikatoren ähnlicher geworden, allerdings nur in geringfügigem Maße. Im Falle von Kompetenzarmut ist die steigende Kon- vergenz zudem kein positiver Ausweis. Sie bedeutet vielmehr eine durchschnittliche Verschlechterung der Lage seit 2000. Divergente Entwicklungen zeigen sich für die europäischen Staaten mit Blick auf Weiterbildung. Von einem gemeinsamen europäischen Bildungsraum kann daher noch keine Rede sein. Wichtige bildungspolitische Voraussetzungen, die die Freizügigkeit von Arbeitnehmerinnen und Arbeitnehmern, Unternehmen und Dienstleistungen unterstützen können, sind bislang nicht gegeben. Ein einheitlicher Bildungsraum, der die gegenseitige Anerkennung von Prüfungsleistungen und -abschlüssen wie auch den gleichberechtigten Zugang zu Bildungsinstitutionen ermöglicht, findet sich am ehesten noch im Bereich der Hochschulbildung, angestoßen durch den Bologna-Prozess. Noch ist die EU angesichts der Defizite bei einzelnen Benchmarks nicht fit genug für die Herausforderungen, die postindustrielle Gesellschaften stellen. Der Europäische Rat hat daher im Mai 2009 einen neuen strategischen Rahmen für die europäische Zusammenarbeit auf dem Gebiet der allgemeinen und beruflichen Bildung mit dem Titel „Education \& Training 2020“ verabschiedet (COM 2009). Die bestehenden Benchmarks sollen im Wesentlichen beibehalten und mit neuen Benchmarks ergänzt werden. Neue Benchmarks sind: ein Anteil von Absolventen des Tertiärbereichs von mindestens $40 \%$ bei den 30 bis 34-Jährigen. Ferner soll der Anteil der Kinder im Alter zwischen vier Jahren und dem gesetzlichen Einschulungsalter, die eine vorschulische Einrichtung besuchen, mindestens $95 \%$ betragen.

Die bildungspolitischen Aktivitäten der OECD und EU fördern die Zusammenarbeit der Mitgliedstaaten. Zudem liefern ihre Bildungsberichterstattungen
Informationen darüber, wo für die Bildungspolitik Veränderungsbedarf und Gestaltungsmöglichkeiten bestehen und welche Fortschritte oder auch Rückschritte mit durchgeführten Bildungsreformen gemacht wurden. Auch der konzentrierte Blick auf die Bildungsarmut ist zu begrüßen und steht nationalen Trends entgegen, welche eher „oben“ ansetzen und auf eine Förderung von Eliten abzielen. Wenngleich die EU kein Mandat hat, steuernd in die einzelnen Länderregierungen einzugreifen, so dürfte allein der systematische Länder vergleichende Blick seine Wirkung nicht gänzlich verfehlen. Allerdings erscheinen die Zielmargen als Durchschnittswerte für Europa zu unambitioniert. Geht es um Fragen sozialer Ungleichheit und gesellschaftlicher Integration, wäre eine Orientierung an Positivbeispielen wie Finnland oder Irland, etwa in Bezug auf geringe Bildungsarmut, künftig wünschenswert. Aspekte sozialer Mobilität fehlen in den Benchmarks für 2010 und auch in den neuen Benchmarks der Europäischen Union für 2020 völlig. Zu begrüßen ist der Stellenwert, den die EU in den neuen Benchmarks der frühkindlichen Bildung einräumt. Aus der Bildungsökonomie wissen wir um deren zentrale Bedeutung für den späteren Bildungserfolg (Cunha et al. 2009).

Da gering Qualifizierte in der Wissensgesellschaft seltener Beschäftigung finden und weil höhere Bildung Produktivitätssteigerungen begünstigt, sind verbesserte Bildungsangebote erforderlich. Die Bemühungen der europäischen Mitgliedstaaten müssen sich noch stärker auf das Ausmaß an Bildungsarmut, kognitiver Ungleichheiten und sozialer Mobilität richten, will sich die europäische Wirtschaft nicht selbst Grenzen des eigenen Wachstums setzen. 


\section{LITERATUR}

Allmendinger, J. (1999): Bildungsarmut: Zur Verschränkung von Bildungs- und Sozialpolitik, in: Soziale Welt 1, S. 35-50

Allmendinger, J./Ebner, C./Nikolai, R. (2010): Education in Europe and the Lisbon Benchmarks, in: Alber, J./Gilbert, N. (Hrsg.): United in Diversity? Comparing Social Models in Europe and America, Oxford, S. 308-327

Becker, P./Primova, R. (2009): Die Europäische Union und die Bildungspolitik, Diskussionspapier der FG 1, 2007/07, Stiftung Wissenschaft und Politik, Berlin

Bektchieva, J. (2004): Die europäische Bildungspolitik nach Maastricht, Münster

Belitz, H./Clemens, M./Gehrke, B./Gornig, M./Legler, H./Leidmann, M. (2008): Wirtschaftsstrukturen und Produktivität im internationalen Vergleich, Studien zum deutschen Innovationssystem 6, Berlin und Hannover

Boli, J./Ramirez, F./Meyer, J.W. (1985): Explaining the Origins and Expansion of Mass Education, in: Comparative Education Review 2, S. 145-170

Commission of the European Communities (COM) (2003): Education and Training 2010. The Success of the Lisbon Strategy Hinges on Urgent Reforms, SEC (2003) 1250, Brüssel

Commission of the European Communities (COM) (2006): Progress towards the Lisbon Objectives in Education and Training, SEC (2006) 639, Brüsse

Commission of the European Communities (COM) (2008): Progress towards the Lisbon Objectives in Education and Training, Indicators and Benchmarks, SEC (2007) 2293, Brüssel

Commission of the European Communities (COM) (2009): Progress towards the Lisbon Objectives in Education and Training, SEC (2009) 1616, Brüssel

Cunha, F./Heckman, J.J./Lochner, L./Masterov , D.V. (2006): Interpreting the Evidence on Life Cycle Skill Formation, in: Hanushek, E.A./Welch, F. (Hrsg.): Handbook of the Economics of Education, Amsterdam, S. 697-812

Dion, D. (2005): The Lisbon Process: A European Odyssey, in: European Journal of Education 3, S. 295-313

European Commission (2008): Employment in Europe 2008, Brüssel
Expertenkommission Forschung und Innovation (EFI) (Hrsg.) (2009): Gutachten zu Forschung, Innovation und technologischer Leistungsfähigkeit 2009, Berlin Hanushek, E. A./Wößmann L. (2008): The Role of Cognitive Skills in Economic Development, Journal of Economic Literature 3, S. 607-668 Martens, K./Weymann, A. (2008): Die Internationalisierung der Bildungspolitik: Konvergenz nationaler Pfade?, in: Hurrelmann, A./Leibfried, S./Martens, K./Mayer, P. (Hrsg.): Zerfasert der Nationalstaat?, Frankfurt, S. 243-272

Müller, W./Steinmann, S./Schneider, R. (1997): Bildung in Europa, in: Stefan, H./Immerfall, S. (Hrsg.): Die Westeuropäischen Gesellschaften im Vergleich, Opladen, S. 177-246

Organisation for Economic Co-operation and Development (OECD) (2001): Knowledge and Skills for Life: First Results from PISA 2000, Paris Organisation for Economic Co-operation and Development (OECD) (2004): Learning for Tomorrow's World: First results from PISA 2003, Paris

Organisation for Economic Co-operation and Development (OECD) (2007): PISA 2006, Science Competencies for Tomorrow's World, Paris Organisation for Economic Co-operation and Development (OECD) (2009): Education at a Glance, Paris

Pépin, L. (2006): The History of European Cooperation in Education and Training: Europe in the Making - An Example, Luxembourg Pépin, L. (2007): The History of EU Cooperation in the Field of Education and Training: How Life-Long Learning Became a Strategic Objective, in: European Journal of Education 1, S. 121-132 Rohrbach, D. (2007): The Development of Knowledge Societies in 19 OECD Countries between 1970 and 2002, in: Social Science Information 4, S. 655-689

Schneider, S. L. (Hrsg.) (2008): The International Standard Classification of Education (ISCED 97). An Evaluation of Content and Criterion Validity for 15 European Countries, Mannheim

United Nations (UN) (2008): World Population Prospects: The 2008 Revision. Population Database, http://esa.un.org/unpp/ (Zugriff am 08.07.2009)

Walkenhorst, H. (2008): Explaining Change in EU Education Policy, in: Journal of European Public Policy 4, S. 567-587 